\title{
Substituent effect on photophysical properties of bi-1,3,4-oxadiazole derivatives in solution
}

\author{
Fangyi Chen, ${ }^{\dagger,}$ a Taiji Tian, ${ }^{\dagger, ~ a}$ Chengxiao Zhao, ${ }^{a}$ Binglian Bai, ${ }^{c}$ Min Li, ${ }^{a}$ Haitao Wang ${ }^{*}, a, b$ \\ ${ }^{\dagger}$ These authors contributed equally to this work \\ * Corresponding author. \\ ${ }^{a}$ Key Laboratory of Automobile Materials (MOE) \& College of Materials Science and Engineering, Jilin University, Changchun 130012, China. \\ E-mail: haitao_wang@jlu.edu.cn \\ ${ }^{b}$ Institute of Theoretical Chemistry, Jilin University, Changchun 130023, China. \\ ${ }^{c}$ College of Physics, Jilin University, Changchun 130012, China
}

Keywords: bi-1, 3, 4-oxadiazole derivatives; photophysical properties; substituent effect; DFT; CAM-B3LYP;

Abstract A series of phenyl substituted bi-1,3,4-oxadiazole derivatives were designed and synthesized; the effect of substituent on the photophysical properties and molecular electronic structure was fully studied by the combination of experimental techniques and theoretical calculations. Compared to parent compound without any substituent (BOXD), fluoro-substituent shows little effect on the absorption and emission spectra, whilst a little larger spectral red-shift could be observed for methoxy-, nitro-substituted derivatives and thienyl-substituted bi-1,3,4-oxadiazole (TBOXD). These spectral changes can be well explained by theoretically calculated HOMO and LUMO energy level changes. All these molecules show high fluorescence quantum yield except for nitro-substituted derivative in dilute solutions. The quantum yield of BOXD changes with the concentration and exhibits a high value at the concentrated solution. This work revealed the influence of substituent on the photophysical properties of bi-1,3,4-oxadizaole derivatives in dilute solutions and provided guidance for designing molecules with potential application.

\section{Introduction}

In recent years, organic fluorescent materials have been studied extensively, not only to explore their fundamental optical and electrical properties, but also to identify their potential applications in optoelectronic field, such as organic light-emitting diodes (OLED),[1-5] organic light-emitting field-effect transistors (OLEFETs),[6-10] organic solid-state lasers[11-15] and organic fluorescent sensors.[16-19] Although there have been a lot of reports focusing on the organic fluorescent materials, many problems are still not solved. For instance, most molecules show a high fluorescence yield in their dilute solutions while exhibit an opposite situation in aggregated or condensed state.[20-22] It is reported that the fluorescence quenching may be caused by certain interactions among the adjacent molecules.[22-24] Moreover, some molecules have high charge carrier mobility for the existence of large extent of $\pi-\pi$ interactions but show a very low fluorescence.[25] It is difficult to achieve the high fluorescence and the high charge carrier mobility simultaneously. The properties of organic light-emitting materials such as fluorescence and charge carrier mobility are not only decided by the molecular electronic structure but also by the packing modes and orientation.[22, 26, 27] How the molecular structure influences the photophysical properties and the mechanism is yet to be clear, which seriously restrict the applications of the organic light-emitting materials. In order to explore these problems, much more systematic research should be done. Thoroughly understanding the substituent effect is the basis to obtain high efficient solid state light-emitting materials and provides guidance for the molecular design of organic light-emitting materials.

Among the various $\pi$-conjugated systems, the phenyl substituted 1,3,4-oxadiazole (OXD) unit is an ideal mode to study the photophysical properties due to their tunable energy gap, high fluorescence quantum yield, good thermal and chemical stabilities.[28-31] However, systematically exploring the influence of substituent on the photophysical properties, the solid state molecular packing, and further more the solid state light-emission 


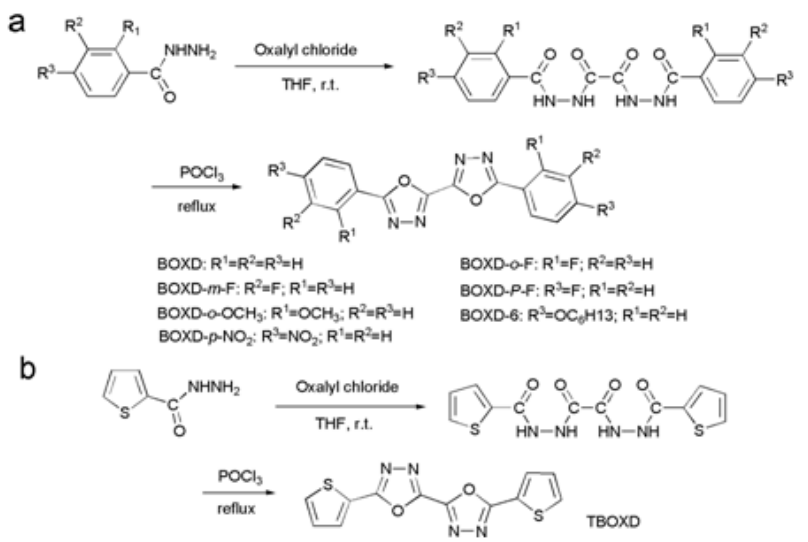

Scheme 1 Synthetic routes to a series of bi-1,3,4-oxadiazole derivatives

properties of 1,3,4-oxadiazole derivatives are still missing. Recently, we have designed and synthesis a series of phenyl substituted bi-1,3,4-oxadiazole (OXD) derivatives, where both the type and the position of the substituents are widely changed (Scheme 1). Here we will focus on the synthesis and photophysical properties of these bi-1, 3, 4-oxadiazole derivatives in dilute solution. In order to get deep insight into the nature of the photophysical properties, experimental study and theoretical calculations were carried out jointly.

\section{Experimental}

\section{Molecular design and Synthesis}

In designing a molecule, various substituents $\left(-\mathrm{C}_{4} \mathrm{H}_{3} \mathrm{~S}\right.$ (thiophene), $-\mathrm{F},-\mathrm{OCH}_{3},-\mathrm{NO}_{2}$ ) with a range of electron-withdrawing and electron-donating properties and different substituted positions (Ortho-, $o-;$ Meta-, $m-$; Para-, $p$-) were selected to assess their effects on these molecules (the molecular structures were shown in Scheme 1). In nomination, $-\mathrm{F},-\mathrm{OCH}_{3}$ and $-\mathrm{NO}_{2}$ indicate the substituents in the phenyl rings, $-o-,-m-$, and $-p$ - indicate the substituted position, for example BOXD- $o$-F indicates that the phenyl ring in BOXD was ortho-substituted by -F; BOXD-6[32] is a molecule that was para-substituted by $-\mathrm{OC}_{6} \mathrm{H}_{13}$ in $\mathrm{BOXD}$; TBOXD represents a thienyl-substituted bi-oxadiazole derivative. All these compounds were obtained through the synthetic route in Scheme 1a, except for TBOXD in Scheme 1b. As shown in Scheme 1, firstly, the oxalyl chloride was slowly dropped into 50 $\mathrm{mL}$ tetrahydrofuran (THF) solution of the corresponding hydrazide derivative. The mixture was stirred at room temperature for about $10 \mathrm{~h}$ and the resulting solution was filtered, yielding oxalyl acid dihydrazide. The crude product was purified by washing with boiling ethanol. Secondly, oxalyl acid dihydrazide was dissolved in $50 \mathrm{~mL}$ phosphorous oxychloride $\left(\mathrm{POCl}_{3}\right)$. The mixture was stirred and heated to reflux for about $40 \mathrm{~h}$. After the reaction, the resulting solution was cooled to room temperature and poured into $1000 \mathrm{~mL}$ ice water. At last, the product was collected by filtration and recrystallized from ethanol or dimethyl sulfoxide (DMSO) for further ${ }^{1} \mathrm{H}$ NMR, FT-IR measurements, and elemental analysis. The original ${ }^{1} \mathrm{H}$ NMR spectra of these bi-oxadiazole derivatives can be found in the supporting information (Figure S1-S7).

\section{2,2-bis(phenyl)-bi-1,3,4-oxadiazole (BOXD)}

Benzoyl hydrazine (3 g, $0.022 \mathrm{~mol}$ ) and oxalyl chloride (1.4 g, 0.011 mol) were added. Yield > $70 \%$, melting point : $273-$ $275{ }^{\circ} \mathrm{C} .{ }^{1} \mathrm{H}$ NMR $(300 \mathrm{MHz}, \mathrm{DMSO}),(\mathrm{ppm}$, from TMS): 8.17 (d, $J=6.82 \mathrm{~Hz}, 2 \mathrm{H}), 7.72(\mathrm{~m}, J=8.04 \mathrm{~Hz}, 3 \mathrm{H})$. FT-IR $(\mathrm{KBr}$, pellet, $\left.\mathrm{cm}^{-1}\right): 3434,3064,2923,2853,2350,1621,1602,1585,1540$, 1476, 1467, 1415, 1399, 1334, 1306, 1286, 1261, 1151, 1081, 1067, 1027, 993, 953, 907, 782, 707, 686. Elemental analysis: Found: C, 66.55; $\mathrm{H}, 3.34 ; \mathrm{N}, 19.33$. Calc. for $\mathrm{C}_{16} \mathrm{H}_{8} \mathrm{~N}_{6} \mathrm{O}_{6}$ : C, 66.20; $\mathrm{H}, 3.47 ; \mathrm{N}, 19.30 \%$

\section{2,2-bis(2-fluorophenyl)-bi-1,3,4-oxadiazole(BOXD-o-F)}

2-Fluorobenzoyl hydrazine (2.43 g, $0.016 \mathrm{~mol})$ and oxalyl chloride $(0.9 \mathrm{~g}, 0.007 \mathrm{~mol})$ were added. Yield $>70 \%$, melting point : $215-$ $217{ }^{\circ} \mathrm{C} .{ }^{1} \mathrm{H}$ NMR (300 MHz, CDCl3), (ppm, from TMS): 8.22 (t, J $=8.12 \mathrm{~Hz}, 1 \mathrm{H}), 7.64(\mathrm{ddd}, \mathrm{J}=7.30,5.07,1.58 \mathrm{~Hz}, 1 \mathrm{H}), 7.35(\mathrm{td}, \mathrm{J}$ $=15.54,8.30 \mathrm{~Hz}, 1 \mathrm{H})$. FT-IR $\left(\mathrm{KBr}\right.$, pellet, $\left.\mathrm{cm}^{-1}\right): 3434,3084,2921$, $1614,1588,1541,1468,1436,1269,1231,1161,1153,1120$, 1062, 1085, 995, 952, 874. Elemental analysis: Found: C, 59.24; H, 2.36; N, 17.33. Calc. for $\mathrm{C}_{16} \mathrm{H}_{8} \mathrm{~F}_{2} \mathrm{~N}_{4} \mathrm{O}_{2}$ : C, 58.90; H, 2.47; $\mathrm{N}$, $17.17 \%$

\section{2,2-bis(3-fluorophenyl)-bi-1,3,4-oxadiazole (BOXD-m-F)}

3-Fluorobenzoyl hydrazine ( $2.45 \mathrm{~g}, 0.016 \mathrm{~mol})$ and oxalyl chloride $(1.0 \mathrm{~g}, 0.008 \mathrm{~mol})$ were added. Yield $>70 \%$, melting point : $214-$ $216{ }^{\circ} \mathrm{C} .{ }^{1} \mathrm{H}$ NMR (300 MHz, DMSO), (ppm, from TMS): 8.01 (dd, $J=17.34,8.42 \mathrm{~Hz}, 2 \mathrm{H}), 7.76(\mathrm{dd}, J=14.03,7.79 \mathrm{~Hz}, 1 \mathrm{H}), 7.62(\mathrm{t}$, $J=8.49 \mathrm{~Hz}, 1 \mathrm{H})$. FT-IR $\left(\mathrm{KBr}\right.$, pellet, $\left.\mathrm{cm}^{-1}\right): 3434,2921,2852$, 2316, 1617, 1593, 1545, 1485, 1466, 1424, 1383, 1335, 1314, 1292, 1274, 1208, 1164, 1145, 1090, 1065, 1010, 1002, 968, 954, 923, 878, 869, 833, 806, 728, 677. Elemental analysis: Found: C, 59.27; $\mathrm{H}, 2.39 ; \mathrm{N}, 17.29$. Calc. for $\mathrm{C}_{16} \mathrm{H}_{8} \mathrm{~F}_{2} \mathrm{~N}_{4} \mathrm{O}_{2}$ : C, 58.90; H, 2.47; $\mathrm{N}, 17.17 \%$

\section{2,2-bis(4-fluorophenyl)-bi-1,3,4-oxadiazole (BOXD-p-F)}

4-Fluorobenzoyl hydrazine (2.44 g, $0.016 \mathrm{~mol}$ ) and oxalyl chloride $(1.0 \mathrm{~g}, 0.008 \mathrm{~mol})$ were added. Yield $>70 \%$, melting point : $275-$ $278{ }^{\circ} \mathrm{C} .{ }^{1} \mathrm{H}$ NMR (300 MHz, DMSO), (ppm, from TMS): 8.24 (dd, $J=8.62,5.38 \mathrm{~Hz}, 2 \mathrm{H}), 7.54(\mathrm{t}, J=8.78 \mathrm{~Hz}, 2 \mathrm{H})$. FT-IR $(\mathrm{KBr}$, 
pellet, $\mathrm{cm}^{-1}$ ): 3427, 2920, 2850, 1608, 1556, 1492, 1476, 1299, 1287, 1225, 1159, 1099, 1083, 1011, 953, 847, 828, 813, 786, 737, 696. Elemental analysis: Found: C, 59.24; H, 2.51; N, 17.25. Calc. for $\mathrm{C}_{16} \mathrm{H}_{8} \mathrm{~F}_{2} \mathrm{~N}_{4} \mathrm{O}_{2}: \mathrm{C}, 58.90 ; \mathrm{H}, 2.47 ; \mathrm{N}, 17.17 \%$

\section{2,2-bis(2-methyloxyphenyl)-bi-1,3,4-oxadiazole} (BOXD-o-OCH${ }_{3}$ )

2-Methoxybenzoyl hydrazine (2.02 g, $0.012 \mathrm{~mol})$ and oxalyl chloride $(0.75 \mathrm{~g}, 0.006 \mathrm{~mol})$ were added. Yield $>70 \%$, melting point :193 - $196{ }^{\circ} \mathrm{C} .{ }^{1} \mathrm{H}$ NMR (300 MHz, DMSO), (ppm, from TMS): 8.00 (dd, $J=7.76,1.67 \mathrm{~Hz}, 1 \mathrm{H}), 7.70$ (ddd, $J=8.95,7.46$, $1.73 \mathrm{~Hz}, 1 \mathrm{H}), 7.35$ (d, $J=8.39 \mathrm{~Hz}, 1 \mathrm{H}), 7.21(\mathrm{t}, J=7.91 \mathrm{~Hz}, 1 \mathrm{H})$, 3.97 (s, 3H). FT-IR (KBr, pellet, $\left.\mathrm{cm}^{-1}\right)$ : 3434, 3084, 2950, 2843, 1604, 1583, 1498, 1473, 1452, 1438, 1328, 1286, 1265, 1184, 1164, 1135, 1081, 1058, 1047, 1010, 949, 857, 798, 777, 767, 749, 704, 671, 583, 533. Elemental analysis: Found: C, 62.01; H, 3.83; $\mathrm{N}, 16.07$. Calc. for $\mathrm{C}_{18} \mathrm{H}_{14} \mathrm{~N}_{4} \mathrm{O}_{4}: \mathrm{C}, 61.71 ; \mathrm{H}, 4.03 ; \mathrm{N}, 15.99 \%$

\section{2,2-bis(4-nitrophenyl)-bi-1,3,4-oxadiazole (BOXD-p-NO ( $_{2}$}

4-Nitrobenzoyl hydrazine $(2.57 \mathrm{~g}, 0.014 \mathrm{~mol})$ and oxalyl chloride $(0.9 \mathrm{~g}, 0.007 \mathrm{~mol})$ were added. Yield > $70 \%$, melting point > $320{ }^{\circ} \mathrm{C} .{ }^{1} \mathrm{H}$ NMR (300 MHz, DMSO), (ppm, from TMS): 8.51 (d, $J=8.67 \mathrm{~Hz}, 2 \mathrm{H}), 8.44$ (d, $J=8.68 \mathrm{~Hz}, 2 \mathrm{H})$. FT-IR (KBr, pellet, $\mathrm{cm}^{-1}$ ): 3434, 3107, 2923, 2853, 2351, 1954, 1609, 1549, 1529, 1498, 1482, 1472, 1413, 1383, 1342, 1313, 1291, 1259, 1161, 1114, 1081, 1012, 998, 954, 868, 855, 761, 713, 673, 666, 626. Elemental analysis: Found: C, 50.92; H, 2.02; N, 22.12. Calc. for $\mathrm{C}_{16} \mathrm{H}_{8} \mathrm{~N}_{6} \mathrm{O}_{6}$ : C, 50.54; H, 2.12; N, 22.10\%

\section{2,2-bis(thienyl)-bi-1,3,4-oxadiazole (TBOXD)}

2-Thiophenhydrazine (2.5 g, $0.018 \mathrm{~mol})$ and oxalyl chloride (1.11 g, 0.009 mol) were added. Yield $>70 \%$, melting point : $268-$ $271{ }^{\circ} \mathrm{C} .{ }^{1} \mathrm{H}$ NMR (300 MHz, DMSO), (ppm, from TMS): 8.09 (d, $J=4.94 \mathrm{~Hz}, 1 \mathrm{H}), 8.05(\mathrm{~s}, J=3.13 \mathrm{~Hz}, 1 \mathrm{H}), 7.38(\mathrm{t}, J=7.12,1 \mathrm{H})$. FT-IR $\left(\mathrm{KBr}\right.$, pellet, $\left.\mathrm{cm}^{-1}\right)$ : 3430, 3114, 2924, 2853, 1732, 1627, $1581,1484,1471,1433,1417,1343,1322,1261,1228,1151$, 1078, 1067, 1032, 953, 940, 862, 852, 803, 751, 723, 669, 654. Elemental analysis: Found: C, 47.31; H, 1.87; N, 18.43; S, 21.26. Calc. for $\mathrm{C}_{12} \mathrm{H}_{6} \mathrm{~N}_{4} \mathrm{O}_{2} \mathrm{~S}_{2}: \mathrm{C}, 47.67 ; \mathrm{H}, 2.00 ; \mathrm{N}, 18.53 ; \mathrm{S}, 21.21 \%$

\section{Characterizations and Computational details}

${ }^{1} \mathrm{H}$ NMR spectra were recorded with a Mercury-300BB $300 \mathrm{MHz}$ spectrometer, using $\mathrm{CDCl}_{3}$ or DMSO as a solvent and tetramethylsilane (TMS) as an internal standard ( $\delta=0.00 \mathrm{ppm})$. UV-vis absorption spectra were recorded on a Shimazu UV-2550 spectrometer, and photoluminescence was measured on a
Perkin-Elmer LS 55 spectrometer. The room-temperature luminescence quantum yields in solutions were determined relative to quinine sulfate acid aqueous solution (0.546) and calculated according to the following equation: $\Phi_{\mathrm{unk}}=\Phi_{\mathrm{std}}$ $\left(I_{\text {unk }} / A_{\text {unk }}\right)\left(A_{\text {std }} / I_{\text {std }}\right)\left(\eta_{\text {unk }} / \eta_{\text {std }}\right)^{2}$, where $\Phi_{\text {unk }}$ is the radiative quantum yield of the sample; $\Phi_{\text {std }}$ is the radiative quantum yield of the standard; $I_{\text {unk }}$ and $I_{\text {std }}$ are the integrated emission intensities of the sample and standard, respectively; $A_{\text {unk }}$ and $A_{\text {std }}$ are the absorptions of the sample and standard at the excitation wavelength respectively; $\eta_{\text {unk }}$ and $\eta_{\text {std }}$ are the indexes of refraction of the sample and standard solutions (pure solvents were assumed), respectively. FTIR spectra were recorded with a Perkin-Elmer spectrometer (Spectrum One B) and the sample was in the form of a pressed tablet with $\mathrm{KBr}$.

In theoretical calculations, the ground state geometry of each molecule was optimized at density functional theory (DFT) level; and the excitation energy of these compounds was calculated at time-dependent DFT level. Considering the charge transfer character might be involved in this system, a range-separated hybrid functional (CAM-B3LYP) was applied. In order to compare with experimental observations, the excitation energy was computed in tetrahydrofuran with a PCM model. All these calculations were carried out with Gaussian 09 software package (version A.02)[33] and the standard $6-31 \mathrm{G}^{* *}$ basis set was employed.

\section{Results and discussion}

\section{Spectroscopic studies}

The photophysical properties of all these compounds were measured in the tetrahydrofuran (THF) solution with the concentration of $1 \times 10^{-5} \mathrm{M}$ except BOXD- $p-\mathrm{NO}_{2}$ in $10^{-6} \mathrm{M}$. As shown in Figure 1a and Table 1, the parent compound BOXD, which is without any substituent, shows an intense absorption at $297 \mathrm{~nm}$. The fluoro-substituted homologues show little changes in absorption spectra compared with BOXD: the absorption maximum $\left(\lambda_{\mathrm{abs}}\right)$ is 295, 297 and $297 \mathrm{~nm}$ for BOXD-o-F, BOXD- $m-\mathrm{F}$, and BOXD-p-F, respectively. The absorption band width of BOXD- $m-\mathrm{F}$, and BOXD- $p-\mathrm{F}$ is equivalent to BOXD, while in the case of BOXD-o-F, it becomes much wider. Nitroand methoxy-substituents have a momentous impact on the absorption spectra. For BOXD- $p-\mathrm{NO}_{2}$, it red-shifts about $17 \mathrm{~nm}$ in wavelength $\left(\lambda_{\mathrm{abs}}=314 \mathrm{~nm}\right)$. For methoxy-substituted derivatives, the para-substituted one (BOXD-6)[32] can make a red-shift by about $23 \mathrm{~nm}$, and ortho-substituted one (BOXD-o-OCH$)$ shows two absorption bands, one is close to $318 \mathrm{~nm}$ and the other one is 
Table 1 Photophysical characteristics of all the bi-1,3,4-oxadiazole derivatives.

\begin{tabular}{ccccc}
\hline Compound & $\lambda_{\mathrm{abs}}(\mathrm{nm})^{\mathrm{a}}$ & $\lambda_{\mathrm{em}}(\mathrm{nm})^{\mathrm{b}}$ & $\lambda_{\mathrm{ex}}(\mathrm{nm})^{\mathrm{c}}$ & $\Phi(\%)^{\mathrm{d}}$ \\
\hline BOXD & 297 & 365 & 305 & 73.5 \\
BOXD- $o-\mathrm{F}$ & 295 & 365 & 303 & 78.9 \\
BOXD- $m-\mathrm{F}$ & 297 & 364 & 303 & 79.8 \\
BOXD- $p-\mathrm{F}$ & 297 & 366 & 303 & 61.9 \\
BOXD- $o-O C \mathrm{H}_{3}$ & 284,318 & 377 & 324 & 92.5 \\
BOXD-6 & 320 & 386 & 320 & 84.6 \\
TBOXD & 322 & 385 & 332 & 74.9 \\
BOXD- $p-\mathrm{NO}_{2}$ & 314 & 395 & 310 & 24.7 \\
\hline
\end{tabular}

a absorption maximum in THF solution $\left(1 \times 10^{-5} \mathrm{M}\right){ }^{\mathrm{b}}$ emission maximum in THF solution $\left(1 \times 10^{-5} \mathrm{M}\right)$ except BOXD-p- $\mathrm{NO}_{2}$ in $10^{-6} \mathrm{M} ;{ }^{\mathrm{c}}$ excitation maximum in THF solution $\left(1 \times 10^{-5} \mathrm{M}\right)$ except BOXD-p- $\mathrm{NO}_{2}$ in $10^{-6} \mathrm{M}$; ${ }^{\mathrm{d}}$ quantum efficiency in THF solution $\left(1 \times 10^{-6} \mathrm{M}\right)$ except BOXD-6 in $5 \times$ $10^{-6} \mathrm{M}$

a)

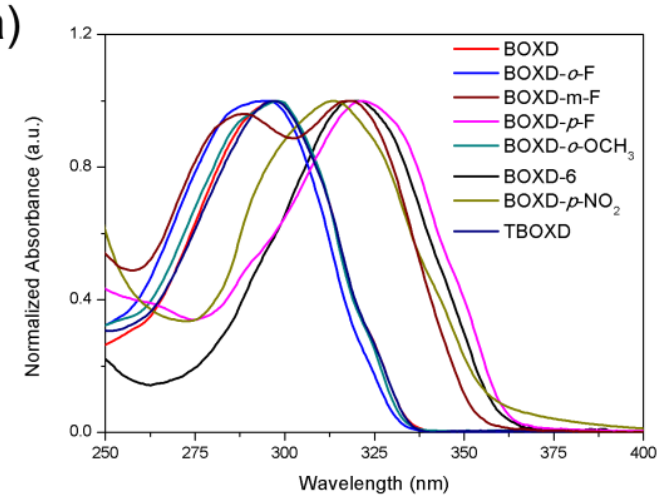

b)

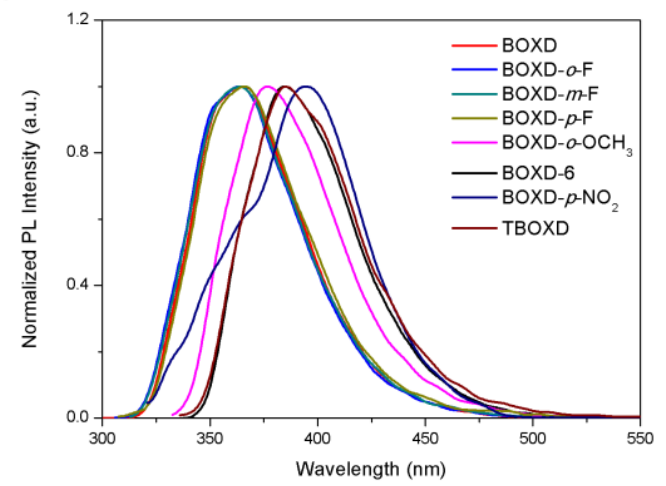

Figure 1 a): Normalized UV-vis absorption spectra of bi-1,3,4-oxadiazole derivatives in THF solution. b): Normalized fluorescence spectra of 1,3,4-oxadiazole derivatives in THF solution.

at $284 \mathrm{~nm}$. Replacing the benzene with a thiophene ring can make a large red-shifts about $25 \mathrm{~nm}\left(\right.$ TBOXD, $\left.\lambda_{\mathrm{abs}}=322 \mathrm{~nm}\right)$. The broad absorption band (BOXD-o-F) or double bands (BOXD-o-OCH ${ }_{3}$ ) observed for these ortho-substituted derivatives may be ascribed to the fact that there is a stable twist conformer besides the planar conformer due to the steric effect. The higher-energy absorption band might be assigned to those molecules with twist conformation, while the lower-energy band is from the planar conformation. This hypothesis can be further confirmed by

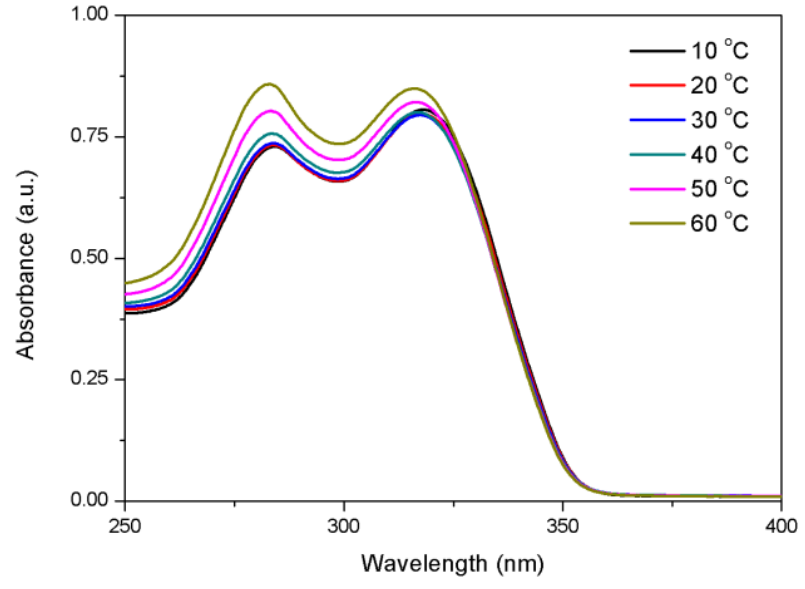

Figure $2 \mathrm{UV}$-vis absorption spectra of BOXD-o- $\mathrm{OCH}_{3}$ in THF solution $\left(\sim 1 \times 10^{-5} \mathrm{M}\right)$ at different temperature

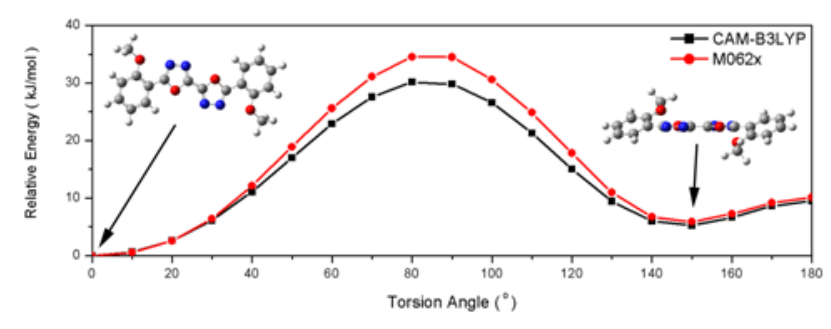

Figure 3 the curve of relative energy and torsion angle of BOXD-o-OCH computed with M062x/6-31G**and CAM-B3LYP/6-31G** methods

variable temperature UV-vis absorption spectroscopic study and theoretical calculations. Figure 2 shows the non-normalized absorption spectra of BOXD-o- $\mathrm{OCH}_{3}$ at variable temperature in THF solution $\left(\sim 10^{-5} \mathrm{M}\right)$ and the normalized result is shown in figure S8. It can be clearly found that the intensity of the two peaks both increased with the temperature increased, but the relative increase in intensity at $284 \mathrm{~nm}$ is bigger than that at 318 $\mathrm{nm}$. It supports our hypothesis that the existence of two different conformers and the planar conformation will gradually change to the twist conformation with the increasing temperature. The theoretical calculation has shown that the global energy minimum conformation of BOXD-o- $\mathrm{OCH}_{3}$ is a planar one (Figure 3). In order to search for a twist conformer as observed in the crystal structure (Figure S9 a), a rigid scan on the twist angle between the benzene ring and the oxadiazole ring has been carried out with both M062x/6-31G**and CAM-B3LYP/6-31G** methods. As shown in Figure 3, the energy goes up with the increasing torsion angle and yields a lowest energy at both $0^{\circ}$ and $150^{\circ}$, confirming the possibility to have two-type conformers in solution. These results could support our hypothesis that the existence of two different conformers is responsible for the feature of double or broader absorption peak in ortho-substituted derivatives. In 
addition, we have tried to growth single crystal in different conditions to obtain different conformers, but so far only the twist conformer in which the dihedral angle between the benzene ring and the oxadiazole ring is $10.53^{\circ}$ has been observed for BOXD-o- $\mathrm{OCH}_{3}$ (Figure S8 a); while planar conformer can be found in other compounds including BOXD-o-F (Figure S9 b).[34, 35]

The fluorescence spectra of these 1,3,4-oxadiazole derivatives are shown in Figure $1 \mathrm{~b}$ and all of them were measured at their maximum excitation wavelength, respectively. BOXD shows an intense emission at $365 \mathrm{~nm}$. Similarly to the absorption spectra, fluoro-substituent also has little effect on the fluorescence emission spectra. The emission maxima $\left(\lambda_{\mathrm{em}}\right)$ are 365,364 and 366 $\mathrm{nm}$ for BOXD- $-\mathrm{F}$, BOXD- $m-\mathrm{F}$ and BOXD- $p-\mathrm{F}$, respectively, which are very close to BOXD $\left(\lambda_{\mathrm{em}}=365 \mathrm{~nm}\right)$. The emission band width also can be retained well in these fluoro-substituted derivatives. Nitro- and methoxy-substituents also have higher impact on the fluorescence emission spectra. For BOXD- $p-\mathrm{NO}_{2}$, it red-shifts about $30 \mathrm{~nm}$ in wavelength $\left(\lambda_{\mathrm{em}}=395 \mathrm{~nm}\right)$. For methoxy-substituted derivatives, it red-shifts about $21 \mathrm{~nm}$ in wavelength for the BOXD-6 $\left(\lambda_{\mathrm{em}}=386 \mathrm{~nm}\right)$ and $12 \mathrm{~nm}$ for BOXD-o- $-\mathrm{OCH}_{3}\left(\lambda_{\mathrm{em}}=377 \mathrm{~nm}\right)$. TBOXD, which replaces the benzene with a thiophene ring, exhibits a $20 \mathrm{~nm}$ red-shifts in its maximum $\left(\lambda_{\mathrm{em}}=385 \mathrm{~nm}\right)$. The maximum emission peaks of all the derivatives in THF dilute solution $\left(\sim 10^{-5} \mathrm{M}\right)$ are summarized in Table 1.

All the derivatives show high quantum yields except nitro-substituted derivative (BOXD- $p-\mathrm{NO}_{2}$ ) which displays a low quantum yields $(24.7 \%)$ in the THF dilute solution $\left(\sim 10^{-6} \mathrm{M}\right)$. The quantum yields are list in Table 1 (The photophysical parameters used in calculation of the quantum yields are summarized in Table S2-S3). The value for the parent compound BOXD is $73.5 \%$. In fluoro-substituted derivatives, quantum yields (BOXD-o-F: $78.9 \%$; BOXD-m-F: $79.8 \%$; BOXD-p-F: $61.9 \%$ ) have a comparable value with BOXD and change with the position of fluoro-substituent. BOXD-o- $\mathrm{OCH}_{3}$ exhibits the highest quantum yield among these compounds ( $\Phi: 92.5 \%)$ while for BOXD-6, the quantum yield is about $84.6 \%$ in $5 \times 10^{-6} \mathrm{M}$ which is only lower than BOXD- $o-\mathrm{OCH}_{3}$. TBOXD shows a moderate value of $74.9 \%$. Thus, the introduction of methoxy-substituents in ortho-position and fluoro-substituents in both ortho- and meta-positions of benzene can significantly improve the fluorescence quantum yield. What's more, quantum yield of BOXD was found depend on the concentration of solution. As figure 4 shows, the quantum yield of BOXD is $73.5 \%$ in $10^{-6} \mathrm{M}$

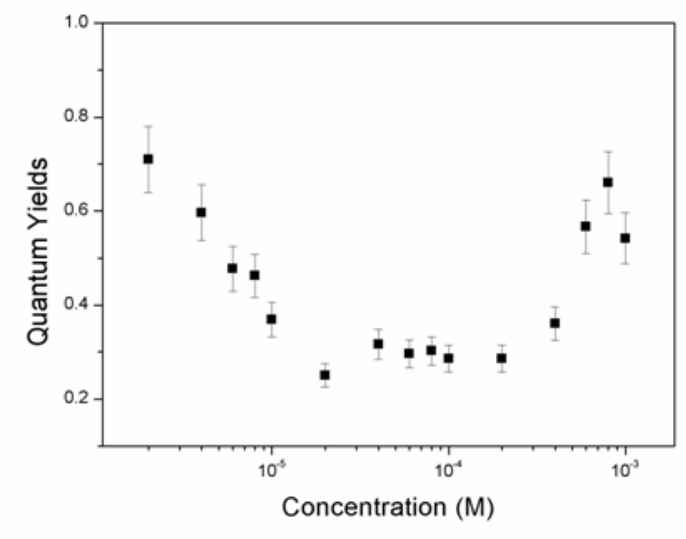

Figure 4 concentration-dependent photoluminescence quantum yields of BOXD in THF

a)

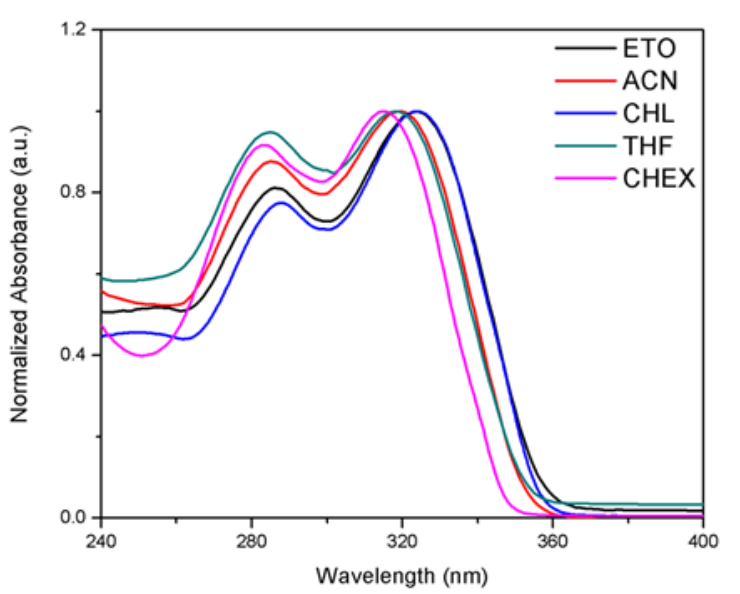

b)

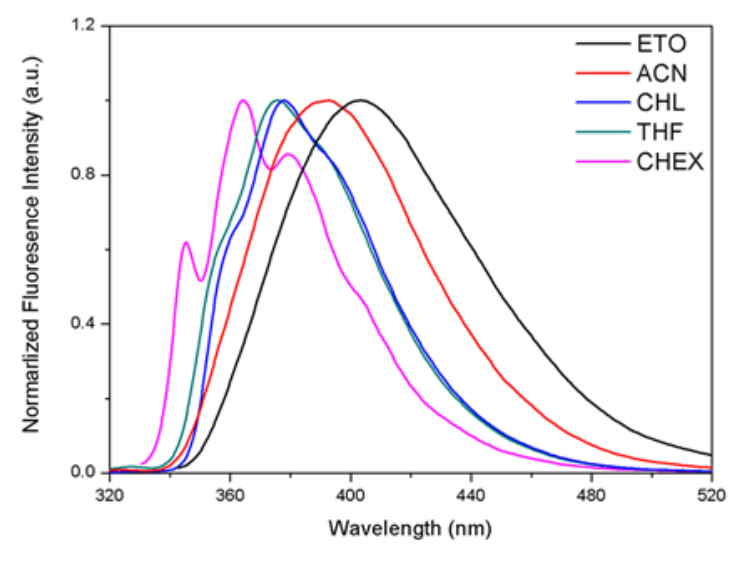

Figure 5 a): UV-vis absorption spectra of BOXD- $o-\mathrm{OCH}_{3}$ (normalized) in different solvents $\left(\sim 1 \times 10^{-5} \mathrm{M}\right)$. b): Fluorescence spectra of BOXD-o-OCH (normalized) in different solvents $\left(1 \times 10^{-5} \mathrm{M}\right)$ (CHEX: cyclohexane; CHL: chloroform; THF: tetrahydrofuran; ACN: acetonitrile; ETO: ethanol).

and rapidly decreased with the concentration increased which could be attributed to the fluorescence quenching caused by the molecular aggregates. The smallest quantum yield is $25.0 \%$ in 
about $2 \times 10^{-5} \mathrm{M}$. This point is obviously smaller than others, which could be caused by the instrumental error. With the concentration continue to increased, the quantum yield gradually increased. When the concentration is about $8 \times 10^{-4} \mathrm{M}$, the quantum yield has the maximum value $(66.0 \%)$ in concentrated solution. This phenomenon is similar to that observed in BOXD-6[36] (which were reported that it is caused by the transition of different type of aggregates in different concentration solution.) The final decrease (from $8 \times 10^{-4}$ to $1 \times 10^{-3} \mathrm{M}$ ) might be due to serious re-absorption of these molecular aggregates observed in the thick solution. BOXD show such interesting concentration-dependent properties and high photoluminescence quantum yields in concentrated solution, which suggests that its possible application as high quantum yield materials in device.

We have also inspected the solvatochromic behavior of BOXD-o- $-\mathrm{OCH}_{3}$ in solvents of different polarity. The absorption and fluorescence emission spectra of BOXD-o-OCH 3 are present in Figure 5. As shown in Figure 5a, the spectrum from non-polar cyclohexane (CHEX) exhibits the maximum absorbance intensity at $315 \mathrm{~nm}$. The ethanol (ETO) spectrum is highly red shift to 323 $\mathrm{nm}$. The spectra in tetrahydrofuran (THF), acetonitrile (ACN) and chloroform (CHL) lie between those in CHEX and ETO. There is only $8 \mathrm{~nm}$ red-shift found indicating that the electronic and structural nature of BOXD-o- $\mathrm{OCH}_{3}$ in the ground state and Franck-Condon excited state, which are responsible for absorption, do not vary much during excitation. The fluorescence spectra of BOXD-o-OCH $\mathrm{OCH}_{3}$ in different solvents are measured at its maximum excitation wavelength respectively. In CHEX, it shows an intense emission at $364 \mathrm{~nm}$ with vibrational fine structure. The fine structure is somewhat visible in solvents with moderate polarity (THF and CHL), but it is totally lost in highly polar solvents (ACN and ETO)). It is found that the emission maxima increase along with increasing of empirical solvent polarity parameter $\mathrm{E}_{\mathrm{T}}^{\mathrm{N}}$ and form linear relationship (figure S10). The emission spectra shows a large red-shift (about $40 \mathrm{~nm}$ ) in their maximum which indicates that intramolecular charge transfer did not occur in the photo excitation process, but occurred during the excited-state molecular geometry relaxation in solutions and reorganization of the solvent molecules. This point has also been demonstrated in our previous work [32]. As the molecular dipole moment (or the local dipole moment in our system) is larger than the ground state, the energy gap between the excited state and the ground state will become narrow, and the maximum emission peak would be red shift.

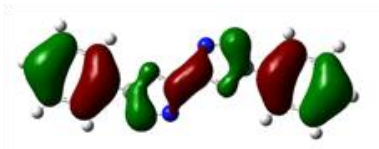

$\mathrm{H}-1$

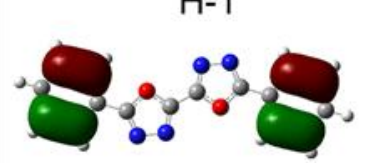

$\mathrm{H}-3$

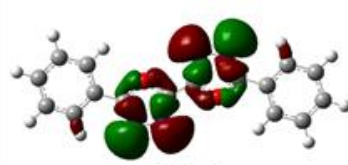

$\mathrm{H}-4$

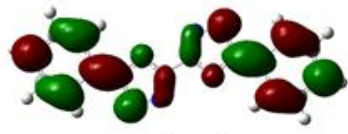

$\mathrm{L}+1$

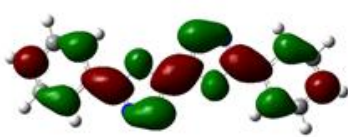

LUMO

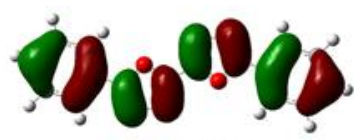

HOMO
Figure 6 Electron density diagrams of molecular orbitals of BOXD computed with CAM-B3LYP/6-31G** method.

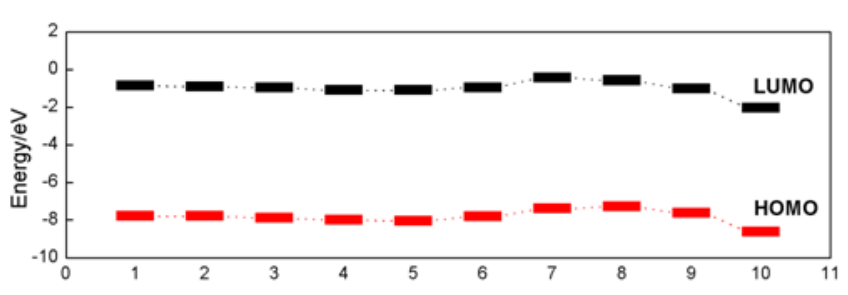

Figure 7 HOMO-LUMO energy states of all the bi-1,3,4-oxadiazole derivatives (1: BOXD; 2: BOXD- $o$-F-1; 3: BOXD- $o-F-2$; 4: BOXD- $m$-F-1; 5: $\quad$ BOXD- $m-\mathrm{F}-2 ; \quad 6: \quad$ BOXD- $p$-F; $\quad 7: \quad$ BOXD- $o-\mathrm{OCH}_{3}-1 ; \quad 8$ : BOXD-o-OCH -2 ; 9: TBOXD; 10: BOXD- $p$ - $\mathrm{NO}_{2}$; Additional numbering (1 and 2) was involved to distinguish two stable conformers found in metaand orth- substituted derivatives: 1 stands that the substituent on the benzene ring is at the same side with the oxygen atom on the oxadiazole ring; 2 stands that the substituent on the benzene ring is at the different side with the oxygen atom on the oxadiazole ring)

\section{Theoretical calculations}

\section{Molecular geometries}

In order to reveal the electronic structure and the natural photophysical properties of these bi-1,3,4-oxadiazole derivatives, theoretical calculations are carried out at the density functional theory level. These calculations predicted planar structure for all these molecules indicating that oxadiazole ring shows great conjugating ability with benzene ring. For the para-substituted derivatives, there is only one stable conformer; while for the ortho- and meta-substituted derivatives, two types of stable conformers could be obtained (Figure S11, denoted as 1 and 2). The energy differences between those different conformers are in the range of 1-8 kJ/mol. As for BOXD-o-F and BOXD-o-OCH the energy of the conformer in which the substituent on the benzene ring is at the same side with the oxygen atom in the oxazole ring (BOXD- $o-\mathrm{F}-1$ and $\mathrm{BOXD}-o-\mathrm{OCH}_{3}-1$ ) is lower than 
Table 2 Excitation energy, oscillator strength and calculated HOMO-LUMO energy states of all the bi-1,3,4-oxadiazole derivatives. ${ }^{a}$

\begin{tabular}{|c|c|c|c|c|c|c|c|c|c|}
\hline \multirow{3}{*}{ Compound } & \multirow{3}{*}{$E_{\mathrm{L}}(\mathrm{eV})$} & \multirow{3}{*}{$E_{H}(\mathrm{eV})$} & \multirow{3}{*}{$\Delta E_{H-L}(\mathrm{eV})$} & \multicolumn{2}{|c|}{ Excitation Energy $(\mathrm{eV})$} & \multirow{2}{*}{$f$} & \multirow{2}{*}{\multicolumn{2}{|c|}{$\begin{array}{c}\text { Main component of } \\
\text { transition }\end{array}$}} & \multirow{2}{*}{$E(\mathrm{~kJ} / \mathrm{mol})$} \\
\hline & & & & \multirow[t]{2}{*}{ Calc. } & \multirow[t]{2}{*}{ Exp. } & & & & \\
\hline & & & & & & & $\mathrm{H}-4 \rightarrow \mathrm{L}$ & $2.1 \%$ & \\
\hline \multirow[t]{3}{*}{ BOXD } & \multirow[t]{3}{*}{-0.87} & \multirow[t]{3}{*}{-7.80} & \multirow[t]{3}{*}{6.93} & \multirow[t]{3}{*}{4.25} & \multirow[t]{3}{*}{4.18} & \multirow[t]{3}{*}{1.35} & $\mathrm{H}-1 \rightarrow \mathrm{L}+1$ & $7.5 \%$ & \multirow[t]{3}{*}{-2584605.1} \\
\hline & & & & & & & $\mathrm{H} \rightarrow \mathrm{L}$ & $88.1 \%$ & \\
\hline & & & & & & & $\mathrm{H}-3 \rightarrow \mathrm{L}+1$ & $2.9 \%$ & \\
\hline \multirow[t]{3}{*}{ BOXD-o-F-1 } & \multirow[t]{3}{*}{-0.91} & \multirow[t]{3}{*}{-7.80} & \multirow[t]{3}{*}{6.90} & \multirow[t]{3}{*}{4.28} & \multirow[t]{3}{*}{4.21} & \multirow[t]{3}{*}{1.41} & $\mathrm{H}-1 \rightarrow \mathrm{L}+1$ & $5.1 \%$ & \multirow[t]{3}{*}{-3105599.7} \\
\hline & & & & & & & $\mathrm{H} \rightarrow \mathrm{L}$ & $87.9 \%$ & \\
\hline & & & & & & & $\mathrm{H}-3 \rightarrow \mathrm{L}+$ & $2.3 \%$ & \\
\hline \multirow[t]{2}{*}{ BOXD-o-F-2 } & \multirow[t]{2}{*}{-0.98} & -7.90 & 6.92 & 4.27 & 4.21 & 1.36 & $\mathrm{H}-1 \rightarrow \mathrm{L}+1$ & $5.7 \%$ & -3105595.7 \\
\hline & & & & & & & $\mathrm{H} \rightarrow \mathrm{L}$ & $87.4 \%$ & \\
\hline BOXD- $m$-F-1 & -1.11 & -8.01 & 6.90 & 4.27 & 418 & 136 & $\mathrm{H}-1 \rightarrow \mathrm{L}+1$ & $6.5 \%$ & -31056162 \\
\hline & & & & & 4.10 & 1.50 & $\mathrm{H} \rightarrow \mathrm{L}$ & $86.2 \%$ & -3105010.2 \\
\hline & & & & & & & $\mathrm{H}-4 \rightarrow \mathrm{L}$ & $2.0 \%$ & \\
\hline BOXD- $m$-F-2 & -111 & -8.05 & 6.94 & 4.29 & 4.18 & 1.34 & $\mathrm{H}-3 \rightarrow \mathrm{L}+1$ & $3.1 \%$ & -3105616.6 \\
\hline (5) & & & & & & & $\mathrm{H}-1 \rightarrow \mathrm{L}+1$ & $4.7 \%$ & \\
\hline & & & & & & & $\mathrm{H} \rightarrow \mathrm{L}$ & $87.3 \%$ & \\
\hline & & & & & & & $\mathrm{H}-4 \rightarrow \mathrm{L}$ & $2.5 \%$ & \\
\hline BOXD- $p-\mathrm{F}$ & -0.96 & -7.82 & 6.86 & 4.24 & 4.18 & 1.34 & $\mathrm{H}-1 \rightarrow \mathrm{L}+1$ & $7.3 \%$ & -3105622.5 \\
\hline & & & & & & & $\mathrm{H} \rightarrow \mathrm{L}$ & $87.8 \%$ & \\
\hline & & & & & & & $\mathrm{H}-2 \rightarrow \mathrm{L}$ & $2.9 \%$ & \\
\hline BOXD-o- $\mathrm{OCH}_{3}-1$ & -0.44 & -7.40 & 6.96 & 4.19 & 4.37 & 0.91 & $\mathrm{H}-1 \rightarrow \mathrm{L}+1$ & $15.0 \%$ & -3185545.0 \\
\hline & & & & & & & $\mathrm{H} \rightarrow \mathrm{L}$ & $75.4 \%$ & \\
\hline & & & & & & & $\mathrm{H}-2 \rightarrow \mathrm{L}$ & $2.9 \%$ & \\
\hline BOXD- $o-\mathrm{OCH}_{3}-2$ & -0.59 & -7.29 & 6.70 & 4.03 & 3.90 & 1.26 & $\mathrm{H}-1 \rightarrow \mathrm{L}+1$ & $12.5 \%$ & -3185538.0 \\
\hline & & & & & & & $\mathrm{H} \rightarrow \mathrm{L}$ & $79.2 \%$ & \\
\hline TBOXD & -102 & $-7,63$ & 661 & 3.97 & 385 & 127 & $\mathrm{H}-1 \rightarrow \mathrm{L}+1$ & $10.8 \%$ & -4269254.5 \\
\hline HDUD & -1.02 & -1.05 & 0.01 & 5. & & & $\mathrm{H} \rightarrow \mathrm{L}$ & $85.5 \%$ & -4201204. \\
\hline & & & & & & & $\mathrm{H}-8 \rightarrow \mathrm{L}+2$ & $2.1 \%$ & \\
\hline BOXD-n-NO & 205 & 862 & 657 & 404 & 305 & 175 & $\mathrm{H}-1 \rightarrow \mathrm{L}+1$ & $14.8 \%$ & 36580671 \\
\hline DUAD-p-1V & $-2.0 J$ & -0.02 & 0.01 & 4.04 & 3.93 & 1.15 & $\mathrm{H} \rightarrow \mathrm{L}$ & $74.3 \%$ & $-505000 / .1$ \\
\hline & & & & & & & $\mathrm{H} \rightarrow \mathrm{L}+2$ & $6.8 \%$ & \\
\hline
\end{tabular}

${ }^{a}$ As for BOXD- $p-\mathrm{NO}_{2}$, electron transitions from the ground state to the first and third singlet excited state are forbidden, so the data presented in the table were the result of electron transition from the ground state to the third single excited state. ${ }^{\mathrm{b}}$ the sum of electronic and zero-point energies in $\mathrm{kJ}^{\cdot} \mathrm{mol}^{-1}$ calculated with CAM-B3LYP/6-31G** method.

those at the different sides (BOXD-o-F-2 and BOXD-o-OCH $3-2$ ); While for BOXD- $m$-F, the two conformers share basically the same energy, indicating that the ortho-substituents might have much more steric effect. The bond length and twist angles in these bi-1,3,4-oxadiazole derivatives do not change a lot (Table S1), which suggests that the different type of substituents have very little influence on the conjugation between benzene and oxadiazole rings.

\section{Frontier orbitals}

Figure 6 presents the electronic density of frontier molecular orbitals of BOXD (others can be obtained in Figure S12). As can be seen, almost all these derivatives share the same characteristics of the HOMO and LUMO orbitals. The HOMO and LUMO orbitals are of $\pi$-bonding and anti-bonding character, respectively. Other occupied orbitals, H-1 H-2, mainly consist of $\pi$-bonding orbitals within each aromatic ring (benzyl and 1,3,4-oxadiazole 
rings); on the contrary, the unoccupied orbitals, $\mathrm{L}+1 \sim \mathrm{L}+2$, are mainly consisted of the $\pi$-antibonding orbitals. In addition, the wave functions are almost delocalized over the whole $\pi$-systems for orbitals HOMO and LUMO, while in other orbitals, the electron density are more or less localized on the 1,3,4-oxadiazole unit or the benzene rings. Table 2 and Figure 7 show the HOMO-LUMO energy levels and energy gap of all these bi-1,3,4-oxadiazole derivatives. When compared with BOXD, fluro-substituent shows little effect on the energy level of these frontier orbitals: there are almost no changes in ortho- and para-substituted derivatives $(0-0.11 \mathrm{eV})$, but a little bit larger change for meta-substituted molecules $(0.21-0.25 \mathrm{eV})$. Nitro-substituted derivative shows a significant decrease in both HOMO and LUMO energies (1.18 eV and $0.82 \mathrm{eV})$. Different from others, methoxy-substituted derivative increases the energy level of both HOMO and LUMO. Since the HOMO and LUMO energy decreased or increased simultaneously, the change of energy gap is very small for fluro- and methoxy-substituted derivatives $(0.07 \mathrm{eV}$ and $0.23 \mathrm{eV})$, while a little bit larger for nitro-substituted derivatives $(0.36 \mathrm{eV})$. For TBOXD, the LUMO energy decreased by $0.15 \mathrm{eV}$ and the HOMO energy increased by $0.17 \mathrm{eV}$, so the energy gap decreased $0.31 \mathrm{eV}$.

\section{Electronic state transitions}

In order to get insight into the excited state properties of these 1,3,4-oxadiazole derivatives, time-dependent density functional theory calculations were also carried out. The data for excitation energy, oscillator strength and composition of the orbital transitions are listed in Table 2. The calculated results reveal that $S_{0}-S_{1}$ transition is highly allowed in almost all the molecules except for nitro-substituted derivative, in which $S_{0}-S_{I}$ transition is forbidden; the mainly allowed transition is $S_{0}-S_{3}$ transition. So the one main peak observed in the UV-vis absorption spectra in BOXD, and fluro- and methoxy-substituted derivatives can be attributed to the transition from ground state to $S_{I}$ state. As compared with the experimental results listed in Table 2, the excitation energy for the first excited by theoretical calculations gave satisfied agreement for the energy difference is within $0.2 \mathrm{eV}$. An inspection of the component orbital transitions makes it clear that the electron transition from the ground state to the first excited state mainly consisted of $\mathrm{H} \rightarrow \mathrm{L}$ transitions, with a little contribution from $\mathrm{H}-1 \rightarrow \mathrm{L}+1$ or other transitions, so the absorption for $S_{l}$ should be mainly assigned as a $\pi-\pi^{*}$ type transition. The $\mathrm{n}-\pi^{*}$ type transition only occupied a small percentage (such as $\mathrm{H}-4 \rightarrow \mathrm{L}$ in BOXD) for most of these molecules. Since the $\Delta E_{H-L}$ is basically the same for all these molecules, it is not surprising to find that there is only small variation observed in the absorption spectra. A little larger change of $\Delta E_{H-L}$ for TBOXD and BOXD-p- $\mathrm{NO}_{2}(\sim 0.3 \mathrm{eV})$ might be the reason for the red shift in absorption spectra.

\section{Conclusion}

A series of bi-1,3,4-oxadiazole derivatives with different substituents were successfully synthesized in a facile rote. Our study shows that the influences of different substituents are different from each other. The molecules with nitro- and methoxy-substituents as well as the thienyl group (TBOXD) exhibit a large red-shift in the absorption and emission maximum compared with BOXD, while the molecules with fluoro-substituent do not show significant influence. All the compounds exhibit very high fluorescence quantum yield in dilute solution except for nitro-substituted molecule. What's more, quantum yield of BOXD changes with the concentration and exhibit a high value at the thick solution. Theoretical calculations can explain the experimental phenomenon very well. According to the calculation results, the excellent fluorescent properties of these bi-1,3,4-oxadiazole derivatives may be attributed to the great rigidity of the phenyl substituted bi-oxadiazole backbones and the highly allowed $\pi-\pi^{*}$ type transition from the ground state to the first singlet excited state. Compared with fluro-substituent, which shows very little influence on the energy levels of HOMO and LUMO, methoxy- and nitro-substituents show a little larger effect, however, the changes of energy gap are still not quite distinct due to the synchronic change of HOMO and LUMO energy. Differently, replacement of the phenyl ring by thiophene ring can decrease the LUMO energy, while increase the HOMO energy so that the energy gap shrink much more obviously. This combinational theoretical and experimental study has revealed the detail mechanism of the substituent effect on the electronic structure and spectra, providing an effective guidance to finely tune the molecular energy levels and energy gap, and further the photophysical properties.

\section{Acknowledgements}

This work was supported by National Science Foundation of China (51103057, 51073071, and 21072076), Postdoctoral Science Foundation of China (2012T50294), Open Project of State Key Laboratory of Supramolecular Structure and Materials (sklssm201501) and Jilin University. 


\section{Notes and References}

Figure S1-S12 and Table S1-S3 are available in the supporting information.

1. J. H. Burroughes; D. D. C. Bradley; A. R. Brown; R. N. Marks; K. Mackay; R. H. Friend; P. L. Burns; A. B. Holmes, Light-emitting diodes based on conjugated polymers. Nature 1990, 347, (6293), 539-541.

2. Arno Kraft; Andrew C. Grimsdale; Andrew B. Holmes, Electroluminescent Conjugated Polymers-Seeing Polymers in a New Light. Angew. Chem. Int. Ed. 1998, 37, (4), 402-428.

$3 . \quad$ R. H. Friend; R. W. Gymer, Electroluminescence in conjugated polymers. Nature 1999, 397, (6715), 121-128.

4. Ullrich Mitschke; Peter BaĖuerle, The electroluminescence of organic materials. J. Mater. Chem 2000, 10, 1471-1507.

5. Andrew C. Grimsdale; Khai Leok Chan; Rainer E. Martin; Pawel G. Jokisz; Andrew B. Holmes, Synthesis Of Light-Emitting Conjugated Polymers For Applications In Electroluminescent Devices. Chem. Rev. 2009, 109, 897-1091.

6. V. Y. Butko; X. Chi; A. P. Ramirez, Free-standing tetracene single crystal field effect transistor. Solid State Communications 2003, 128, (11), 431-434.

7. Mang Mang Ling; Zhenan Bao, Thin Film Deposition, Patterning, and Printing in Organic Thin Film Transistors. Chem. Mater. 2004, 16, 4824-4840.

8. R. W. I. de Boer; M. E. Gershenson; A. F. Morpurgo; V. Podzorov, Organic single-crystal field-effect transistors. phys. stat. sol. (a) 2004, 201, (6), 1302-1331.

9. Marta Mas-Torrent; Concepció Rovira, Role of Molecular Order and Solid-State Structure in Organic Field-Effect Transistors. Chem. Rev. 2011, 111, (8), 4833-4856.

10. Chengliang Wang; Huanli Dong; Wenping Hu; Yunqi Liu; Daoben Zhu, Semiconducting $\pi$-Conjugated Systems in Field-Effect Transistors: A Material Odyssey of Organic Electronics. Chem. Rev. 2012, 112, 2208-2267.

11. G Kranzelbinder; G Leising, Organic solid-state lasers. Rep. Prog. Phys. 2000, 63, (5), 729-762.

12. Michael D. McGehee; Alan J. Heeger, Semiconducting (Conjugated) Polymers as Materials for Solid-State Lasers. Adv. Mater. 2000, 12, (22), 1655-1668.

13. U. Scherf; S. Riechel; U. Lemmer; R. F. Mahrt, Conjugated polymers: lasing and stimulated emission. Current Opinion in Solid State and Materials Science 2001, 5, 143-154.

14. G. Kranzelbinder; E. Toussaere; J. Zyss; A. Pogantsch; E. W. J. List; H. Tillmann; H. H. Hörhold, Optically written solid-state lasers with broadly tunable mode emission based on improved poly (2,5-dialkoxy-phenylene-vinylene). Appl Phys Lett 2002, 80, (5), 716-718.

15. I. D. W. Samuel; G. A. Turnbull, Organic Semiconductor Lasers. Chem. Rev. 2007, 107, (4), 1272-1295.

16. Zhijun Ning; Zhao Chen; Qiong Zhang; Yongli Yan; Shixiong Qian; Yong Cao; He Tian, Aggregation-induced Emission (AIE)-active Starburst Triarylamine Fluorophores as Potential Non-doped Red Emitters for Organic Light-emitting Diodes and Cl2 Gas Chemodosimeter. Adv. Funct. Mater. 2007, 17, (18), 3799-3807.
17. Ling Zang; Yanke Che; Jeffrey S. Moore, One-dimensional self-assembly of planar pi-conjugated molecules: adaptable building blocks for organic nanodevices. Acc Chem Res 2008, 41, (12), 1596-1608.

18. Yanke Che; Ling Zang, Enhanced fluorescence sensing of amine vapor based on ultrathin nanofibers. Chem. Сотmun. 2009, 34, (34), 5106-5108.

19. Dongpeng Yan; Jun Lu; Jing Ma; Min Wei; DavidG. Evans; Xue Duan, Reversibly Thermochromic, Fluorescent Ultrathin Films with a Supramolecular Architecture $\uparrow$. Angew. Chem. Int. Ed. 2011, 50, (3), 720-723.

20. Andreas Dreuw; Jurgen Plotner; Lisa Lorenz; Josef Wachtveitl; Juste E. Djanhan; Jurgen Bruning; Thomas Metz; Michael Bolte; Martin U. Schmidt, Molecular Mechanism of the Solid-State Fluorescence Behavior of the Organic Pigment Yellow 101 and Its Derivatives. Angew. Chem. Int. Ed. 2005, 44, (47), 7783-7786.

21. Brynna J. Laughlin; Tyler L. Duniho; Samantha J. El Homsi; Benjamin E. Levy; Nihal Deligonul; Joshua R. Gaffen; John D. Protasiewicz; Andrew G. Tennyson; Rhett C. Smith, Comparison of 1,4-distyrylfluorene and 1,4-distyrylbenzene analogues: synthesis, structure, electrochemistry and photophysics. Org. Biomol. Chem. 2013, 11, (33), 5425-5434.

22. Sanjoy Mukherjee; Pakkirisamy Thilagar, Design Aspects of Luminescent Organic Crystals. Proc. Natl. Acad. Sci, India. Sect. A: Phys. Sci. 2014, 84, (2), 131-149.

23. Edwin A. Chandross; Carol J. Dempster, Intramolecular excimer formation and fluorescence quenching in dinaphthylalkanes. J. Am. Chem. Soc. 1970, 92, (12), 3586-3593.

$24 . \quad$ D. T. Cramb; S. C. Beck, Fluorescence quenching mechanisms in micelles: the effect of high quencher concentration. Photochemistry and Photobiology A: Chemistry 2000, 134, 87-95.

25. Jerome Cornil; David Beljonne; Jean-Philippe Calbert; Jean-Luc Bredas, Interchain Interactions in Organic p-Conjugated Materials: Impact on Electronic Structure, Optical Response, and Charge Transport. Adv. Mater. 2001, 13, (14), 1053-1067.

26. Baoli Dong; Mingliang Wang; Chunxiang Xu; Qi Feng; Yan Wang, Tuning Solid-State Fluorescence of a Twisted $\pi$-Conjugated Molecule by Regulating the Arrangement of Anthracene Fluorophores. Cryst. Growth Des. 2012, 12, (12), 5986-5993.

27. Qi Feng; Mingliang Wang; Baoli Dong; Chunxiang Xu; Jing Zhao; Hongjuan Zhang, Tuning solid-state fluorescence of pyrene derivatives via a cocrystal strategy. CrystEngComm 2013, 15, (18), 3623-3629

28. Burkhard Schulz; Maria Bruma; Ludwig Brehmer, Aromatic Poly(1, 3, 4-Oxadiazoe)s as Advanced materials. Adv Mater 1997, 9, (8), 601-613.

29. Mukundan Thelakkat; Hans-Werner Schmidt, Low molecular weight and polymeric heterocyclics as electron transport hole-blocking materials in organic light-emitting diodes. Polym. Adv. Technol. 1998, 9, (7), 429-442.

30. Gregory Hughes; Martin R. Bryce, Electron-transporting materials for organic electroluminescent and electrophosphorescent devices. J Mater Chem 2004, 1, 94-107. Songnan Qu; Qipeng Lu; Shaohang Wu; Lijun Wang; 
Xingyuan Liu, Two dimensional directed - Interactions in a linear shaped bi-1,3,4-oxadiazole derivative to achieve organic single crystal with highly polarized fluorescence and amplified spontaneous emissions. J. Mater. Chem. 2012, 22, 24605-24609.

Haitao Wang; Huimin Liu; Fu-Quan Bai; Songnan Qu; Xiaoshi Jia; Xia Ran; Fangyi Chen; Binglian Bai; Chengxiao Zhao; Zibai Liu; Hong-Xing Zhang; Min Li, Theoretical and experimental study on intramolecular charge-transfer in symmetric bi-1,3,4-oxadiazole derivatives. Journal of Photochemistry and Photobiology A: Chemistry 2015, 312, 20-27.

33. M. J. Frisch, G. W. T., H. B. Schlegel, G. E. Scuseria, M. A. Robb, J. R. Cheeseman, G. Scalmani, V. Barone, B. Mennucci, G. A. Petersson, H. Nakatsuji, M. Caricato, X. Li, H. P. Hratchian, A. F. Izmaylov, J. Bloino, G. Zheng, J. L. Sonnenberg, M. Hada, M. Ehara, K. Toyota, R. Fukuda, J. Hasegawa, M. Ishida, T. Nakajima, Y. Honda, O. Kitao, H. Nakai, T. Vreven, J. A. Montgomery, Jr., J. E. Peralta, F. Ogliaro, M. Bearpark, J. J. Heyd, E. Brothers, K. N. Kudin, V. N. Staroverov, R. Kobayashi, J. Normand, K. Raghavachari, A. Rendell, J. C. Burant, S. S. Iyengar, J. Tomasi, M. Cossi, N. Rega, J. M. Millam, M. Klene, J. E. Knox, J. B. Cross, V. Bakken, C. Adamo, J. Jaramillo, R. Gomperts, R. E. Stratmann,
O. Yazyev, A. J. Austin, R. Cammi, C. Pomelli, J. W. Ochterski, R. L. Martin, K. Morokuma, V. G. Zakrzewski, G. A. Voth, P. Salvador, J. J. Dannenberg, S. Dapprich, A. D. Daniels, Ö. Farkas, J. B. Foresman, J. V. Ortiz, J. Cioslowski, and D. J. Fox, Gaussian, Inc., Wallingford CT, 2009. H. Wang; X. Jia; S. Qu; B. Bai; M. Li, 5,5'-Bis(naphthalen-2-yl)-2,2'-bi(1,3,4-oxadiazole). Acta crystallographica. Section E, Structure reports online 2011, 67, (Pt 12), o3360.

35. Songnan Qu; Xiaofang Chen; Xiang Shao; Fan Li; Hongyu Zhang; Haitao Wang; Peng Zhang; Zhixin Yu; Kai Wu; Yue Wang; Min Li, Self-assembly of highly luminescent bi-1,3,4-oxadiazole derivatives through electron donor-acceptor interactions in three-dimensional crystals, two-dimensional layers and mesophases. Journal of Materials Chemistry 2008, 18, (33), 3954.

36. Haitao Wang; Fangyi Chen; Xiaoshi Jia; Huimin Liu; Xia Ran; Mahesh Kumar Ravva; Fu-Quan Bai; Songnan Qu; Min Li; Hong-Xing Zhang; Jean-Luc Brédas, Controllable molecular aggregation and fluorescence properties of 1,3,4-oxadiazole derivatives. J. Mater. Chem. C 2015, 3, (44), 11681-11688. 